\title{
WEAK ASYMPTOTIC HOMOMORPHISM PROPERTY FOR MASAS IN SEMIFINITE FACTORS
}

\author{
KunAL K. MukHERJEe
}

Abstract. The notion of weak asymptotic homomorphism property for masas in semifinite factors is defined and is shown to be equivalent to singularity. The analysis shows that weak asymptotic homomorphism property is a 'spectral phenomenon'.

Mathematics subject classification (2010): 46L10.

Keywords and phrases: von Neumann algebras, semifinite, masa, WAHP.

\section{REFERENCES}

[1] Charles A. Akemann And David Sherman, Conditional expectation onto maximal abelian *subalgebras, preprint: arXiv: 0906.1831, 2010.

[2] J. T. Chang And D. Pollard, Conditioning as disintegration, Statist. Neerlandica 51, 1 (1997), 287-317.

[3] J. DixMIER, Sous-anneaux abéliens maximaux dans les facteurs de type fini, Ann. of Math. (2) 59, (1954), 279-286.

[4] Kenneth J. Dykema, Allan M. Sinclair, And Roger R. Smith, Values of the Pukánszky invariant in free group factors and the hyperfinite factor, J. Funct. Anal. 240, 2 (2006), 373-398.

[5] Junsheng Fang, Mingchu Gao, And Roger R. Smith, The relative weak asymptotic homomorphsim property for inclusions of finite von neumann algebras, Internat. J. Math. 22, 7 (2011), 991-1011.

[6] Junsheng Fang, Roger R. Smith, Stuart A. White, and Alan D. Wiggins, Groupoid normalizers of tensor products, J. Funct. Anal. 258, 1 (2010), 20-49.

[7] RichaRd V. KADISON AND JOHN R. RingROSE, Fundamentals of the theory of operator algebras. Vol. II, American Mathematical Society, volume 16, Providence, RI, 1997.

[8] Roberto Longo, Maximal abelian subalgebras with simple normalizer, Proc. Amer. Math. Soc. 107, 1 (1989), 165-168.

[9] Kunal MukherJee, Masas and Bimodule Decompositions of $\mathrm{II}_{1}$ Factors, Q.J. Math. 62, 2 (2011), 451-486.

[10] SoRIN Popa, Notes on Cartan subalgebras in type $\mathrm{II}_{1}$ factors, Math. Scand. 57, 1 (1985), 171-188.

[11] Sorin Popa And Dimitri Shlyakhtenko, Cartan subalgebras and bimodule decompositions of $\mathrm{II}_{1}$ factors, Math. Scand. 92, 1 (2003), 93-102.

[12] C. E. Silva, Invitation to ergodic theory, American Mathematical Society, volume 42, Providence, RI, 2008.

[13] Allan M. Sinclair And Roger R. Smith, Finite von Neumann algebras and masas, London Mathematical Society Lecture Note Series, volume 351, Cambridge University Press, Cambridge, 2008.

[14] Allan M. Sinclair, Roger R. Smith, Stuart A. White, and Alan Wiggins, Strong singularity of singular masas in $\mathrm{II}_{1}$ factors, Illinois J. Math., 51, 4 (2007), 1077-1084.

[15] Şerban Strătilă, Modular theory in operator algebras, Editura Academiei Republicii Socialiste România, Bucharest, 1981. 